\title{
The Relative Rank of Transformation Semigroups with Restricted Range on a Finite Chain
}

\author{
Kittisak Tinpun \\ Department of Mathematics and Computer Science, Faculty of Science and Technology, Prince of Songkla University, \\ Pattani Campus, Pattani 94000, Thailand
}

Received May 13, 2021; Revised August 5, 2021; Accepted August 22, 2021

Cite This Paper in the following Citation Styles

(a): [1] Kittisak Tinpun, "The Relative Rank of Transformation Semigroups with Restricted Range on a Finite Chain," Mathematics and Statistics, Vol.9, No.5, pp. 718-723, 2021. DOI: 10.13189/ms.2021.090511

(b): Kittisak Tinpun, (2021). The Relative Rank of Transformation Semigroups with Restricted Range on a Finite Chain. Mathematics and Statistics, 9(5), 718-723. DOI: 10.13189/ms.2021.090511

Copyright $\odot 2021$ by authors, all rights reserved. Authors agree that this article remains permanently open access under the terms of the Creative Commons Attribution License 4.0 International License

Abstract Let $S$ be a semigroup and let $G$ be a subset of $S$. A set $G$ is a generating set $G$ of $S$ which is denoted by $\langle G\rangle=S$. The rank of $S$ is the minimal size or the minimal cardinality of a generating set of $S$, i.e. $\operatorname{rank} S:=\min \{|G|: G \subseteq S,\langle G\rangle=S\}$. In last twenty years, the rank of semigroups is worldwide studied by many researchers. Then it lead to a new definition of $\mathrm{r}$ ank that is called the relative rank of $S$ modulo $U$ is the minimal size of a subset $G \subseteq S$ such that $G \cup U$ generates $S$, i.e. $\operatorname{rank}(S: U):=\min \{|G|: G \subseteq S,\langle G \cup U\rangle=S\}$. A set $G \subseteq S$ with $\langle G \cup U\rangle=S$ is called generating set of $S$ modulo $U$. The idea of the relative rank was generalized from the concept of the rank of a semigroup and it was firstly i ntroduced b y H owie, R uškuc a nd $\mathrm{H}$ iggins i n 1998. Let $X$ be a finite chain and let $Y$ be a subchain of $X$. We denote $\mathcal{T}(X)$ the semigroup of full transformations on $X$ under the composition of functions. Let $\mathcal{T}(X, Y)$ be the set of all transformations from $X$ to $Y$ which is so-called the transformation semigroup with restricted range $Y$. It was firstly i ntroduced a nd s tudied b y S ymons i 1 975. Many results in $\mathcal{T}(X)$ were extended to results in $\mathcal{T}(X, Y)$. In this paper, we focus on the relative rank of semigroup $\mathcal{T}(X, Y)$ and the semigroup $\mathcal{O P}(X, Y)$ of all orientation-preserving transformations in $\mathcal{T}(X, Y)$. In Section 2.1, we determine the relative rank of $\mathcal{T}(X, Y)$ modulo the semigroup $\mathcal{O D}(X, Y)$ of all order-preserving or order-reversing transformations. In Section 2.2, we describe the results of the relative rank of $\mathcal{T}(X, Y)$ modulo the semigroup $\mathcal{O P}(X, Y)$. In Section 2.3, we determine the relative rank of $\mathcal{T}(X, Y)$ modulo the semigroup $\mathcal{O P R}(X, Y)$ of all orientation-preserving or orientation-reversing transformations. Moreover, we obtain that the relative rank $\mathcal{T}(X, Y)$ modulo $\mathcal{O P}(X, Y)$ and modulo
$\mathcal{O P} \mathcal{R}(X, Y)$ are equal.

Keywords Generating Set, Transformations, Relative Rank, Orientation-preserving, Orientation-reversing

\section{Introduction and Preliminaries}

Let $X$ be a finite chain, i.e. $X=\{1<\cdots<n\}$ where $n \in \mathbb{N}$. Denote $\mathcal{T}(X)$ by a semigroup of all full transformations on $X$ under the composition of functions. In this paper, we will write functions from the right and compose from the left to the right, i.e. $x(\alpha \beta)=(x \alpha) \beta$. Let $\alpha \in \mathcal{T}(X)$. Define $i m \alpha, \operatorname{rank} \alpha$ and $\operatorname{ker} \alpha$ by $i m \alpha:=\{x \alpha: x \in X\}$, rank $\alpha:=|i m \alpha|$ and $\operatorname{ker} \alpha:=\{(x, y) \in X \times X: x \alpha=y \alpha\}$, respectively. Then $\operatorname{ker} \alpha$ is an equivalence relation on $X$ is called ker $\alpha$-classes. Let $T \subseteq X$ with $|C \cap T|=1$ for all $\operatorname{ker} \alpha$-classes $C$. Then $T$ is a transversal of $\operatorname{ker} \alpha$. For sets $A_{1}, A_{2} \subseteq X$, we write $A_{1}<A_{2}$ if $x_{1}<x_{2}$ for all $x_{1} \in A_{1}$ and for all $x_{2} \in A_{2}$. Let $A \subseteq X$. Define $\left.\alpha\right|_{A}$ by a mapping $\left.\alpha\right|_{A}: A \rightarrow X$ with $x\left(\left.\alpha\right|_{A}\right):=x \alpha$ for all $x \in A$, i.e. $\left.\alpha\right|_{A}$ is the mapping $\alpha$ restricted to $A$.

A set $G$ is a generating set $G$ of a semigroup $S$ which is denoted by $\langle G\rangle=S$. Define the rank of $S$ by a minimal size of a generating set of $S$, i.e. $\operatorname{rank} S:=\min \{|G|: G \subseteq$ $S,\langle G\rangle=S\}$. The relative rank of $S$ modulo $U$ is the minimal size of a subset $G \subseteq S$ such that $G \cup U$ generates $S$, i.e. $\operatorname{rank}(S: U):=\min \{|G|: G \subseteq S,\langle G \cup U\rangle=S\}$. A set $G \subseteq S$ with $\langle G \cup U\rangle=S$ is called generating set of $S$ modulo $U$. The concept of a relative rank generalizes the concept rank of a semigroup and was introduced by Howie, Ruškuc and Higgins [13]. 
In this paper, we also consider an orientation-preserving transformations with a linear order on $X$. Let $\alpha \in \mathcal{T}(X)$. Then is called an orientation-preserving (orientation-reversing, respectively) if there is a decomposition $X=A_{1} \cup A_{2}$ with $A_{1}<A_{2}, y_{1} \alpha \geq y_{2} \alpha\left(y_{1} \alpha \leq y_{2} \alpha\right.$, respectively) for all $y_{1} \in A_{1}$ and $y_{2} \in A_{2}$, and $x \alpha \leq y \alpha(x \alpha \geq y \alpha$, respectively) for all $x \leq y \in A_{1}$ or $x \leq y \in A_{2}$. If $A_{2}=\emptyset$ then $\alpha$ is an order-preserving transformation. Moreover, if $A_{1}=\emptyset$ with $x \alpha \geq y \alpha$ for all $x \leq y \in A_{2}$ then $\alpha$ is an order-reversing transformation. The notation of an orientation-preserving transformation was first studied by McAlister [14] and generalized by Catarino and Higgins [2]. The definition of an orientation-preserving transformation which is given by them is equivalent to that one given in this paper. Notice that the product of two orientation-preserving transformations is an orientation-preserving. Let $\mathcal{O}(X)$ be the semigroup of all order-preserving transformations on $X$, let $\mathcal{O D}(X)$ be the semigroup of all order-preserving or orderreversing transformations on $X$, let $\mathcal{O P}(X)$ be the semigroup of all orientation-preserving transformations on $X$, let $\mathcal{O R}(X)$ be the set of all orientation-reversing transformations on $X$ and let $\mathcal{O P R}(X):=\mathcal{O P}(X) \cup \mathcal{O} \mathcal{R}(X)$ be the semigroup of all orientation-preserving or orientation-reversing transformations on $X$, respectively. Clearly, $\mathcal{O}(X) \subseteq \mathcal{O P}(X)$ and $\mathcal{O}(X) \subseteq \mathcal{O P R}(X)$. It is a proper subsemigroup of $\mathcal{O P}(X)$ and $\mathcal{O P R}(X)$ where $n \geq 2$. The semigroup $\mathcal{O P}(X)$ has been widely investigated (see [1], [2], [4], [6], [9], [16]). In particular, the rank of $\mathcal{O P}(X)$ is 2 [1], the rank of $\mathcal{O}(X)$ is $n$ [6] and the rank of $\mathcal{T}(X)$ is 3 [13]. On the other hand, we have $\operatorname{rank}(\mathcal{T}(X): \mathcal{O}(X))=2$ [13], $\operatorname{rank}(\mathcal{T}(X): \mathcal{O P}(X))=1$ [13] and $\operatorname{rank}(\mathcal{O P}(X): \mathcal{O}(X))=1$ [2].

Let $Y$ be a subchain of $X$, i.e. $Y=\left\{l_{1}<\cdots<l_{m}\right\}$ where $m \in\{1, \ldots, n\}$. Define the following sets by

$$
\begin{aligned}
\mathcal{T}(X, Y) & :=\{\alpha \in \mathcal{T}(X): \operatorname{im} \alpha \subseteq Y\}, \\
\mathcal{O}(X, Y) & :=\{\alpha \in \mathcal{O}(X): \operatorname{im} \alpha \subseteq Y\}, \\
\mathcal{O} \mathcal{D}(X, Y) & :=\{\alpha \in \mathcal{O} \mathcal{D}(X): \operatorname{im} \alpha \subseteq Y\}, \\
\mathcal{O} \mathcal{P}(X, Y) & :=\{\alpha \in \mathcal{O P}(X): \operatorname{im} \alpha \subseteq Y\}, \\
\mathcal{O P} \mathcal{R}(X, Y): & =\{\alpha \in \mathcal{O P} \mathcal{P}(X): \operatorname{im} \alpha \subseteq Y\} .
\end{aligned}
$$

All of them form subsemigroups of $\mathcal{T}(X)$. A semigroup $\mathcal{T}(X, Y)$ is called the full transformation semigroup with restricted range and it is defined by Symons [15]. The other semigroups were introduced by Fernandes et al in [8] and [9], respectively. Transformation semigroups with restricted range have been widely investigated (see [7], [8], [10], [14]). The stirling number of second kind $S(n, m)$ is the rank of $\mathcal{T}(X, Y)$ [12]. In [9], it was shown that $\operatorname{rank}(\mathcal{O P}(X, Y))=\left(\begin{array}{c}n \\ m\end{array}\right)$. In [8], the authors proved that $\operatorname{rank}(\mathcal{O}(X, Y))=\left(\begin{array}{c}n-1 \\ m-1\end{array}\right)+$ $\left|R^{\sharp}\right|$, where $R^{\sharp}$ is the set of captive elements. In [16], Tinpun and Koppitz have already shown $\operatorname{rank}(\mathcal{T}(X, Y): \mathcal{O}(X, Y)$ is equal to $S(n, m)-\left(\begin{array}{c}n-1 \\ m-1\end{array}\right)$ or $S(n, m)-\left(\begin{array}{c}n-1 \\ m-1\end{array}\right)+1$ which depends on the given set $Y$.

The purpose of this paper is to determined the relative rank of $\mathcal{T}(X, Y)$ modulo various subsemigroups. Firstly, the rela- tive rank of $\mathcal{T}(X, Y)$ modulo $\mathcal{O D}(X, Y)$ will be determined. Moreover, we also study and describe the relative rank of $\mathcal{T}(X, Y)$ modulo $\mathcal{O P}(X, Y)$. Finally, we determine the relative rank of $\mathcal{T}(X, Y)$ modulo $\mathcal{O P R}(X, Y)$ which is equal to modulo $\mathcal{O P}(X, Y)$.

\section{Main Results}

\section{$2.1 \operatorname{Rank}(\mathcal{T}(X, Y): \mathcal{O D}(X, Y))$}

In this section, we compute the relative rank of $\mathcal{T}(X, Y)$ modulo $\mathcal{O D}(X, Y)$. Define the set $\mathcal{K}$ by

$$
\begin{gathered}
\mathcal{K}:=\{\operatorname{ker} \phi: \phi \in \mathcal{T}(X, Y), \operatorname{rank} \phi=m\} \backslash\{\operatorname{ker} \phi: \phi \in \\
\mathcal{O}(X, Y), \operatorname{rank} \phi=m\} .
\end{gathered}
$$

Notice that $\{\operatorname{ker} \phi: \phi \in \mathcal{O}(X, Y)\}=\{\operatorname{ker} \phi: \phi \in$ $\mathcal{O D}(X, Y)\}$. Then we have the cardinality of $\mathcal{K}$ as the following lemma.

Lemma 2.1. $[16]|\mathcal{K}|=S(n, m)-\left(\begin{array}{c}n-1 \\ m-1\end{array}\right)$.

For each $K \in \mathcal{K}$, we choose $\alpha_{K} \in \mathcal{T}(X, Y)$ with $i m \alpha_{K}=Y$ and $\operatorname{ker} \alpha_{K}=K$. Hence, $\left|\left\{\alpha_{K}: K \in \mathcal{K}\right\}\right|=$ $S(n, m)-\left(\begin{array}{c}n-1 \\ m-1\end{array}\right)$. Then for each $s \in \mathcal{S}(Y)$ there exists $\mu_{s} \in \mathcal{T}(X, Y) \backslash \mathcal{O}(X, Y)$ with $\left.\mu_{s}\right|_{Y}=s$ and $s$ is not an identity mapping on $Y$.

Lemma 2.2. [16] If $\mathcal{S} \subseteq \mathcal{S}(Y)$ and $\langle\mathcal{S}\rangle=\mathcal{S}(Y)$ then

$$
\mathcal{T}(X, Y)=\left\langle\mathcal{O}(X, Y),\left\{\mu_{s}: s \in \mathcal{S}\right\},\left\{\alpha_{K}: K \in \mathcal{K}\right\}\right\rangle .
$$

In [16], the authors defined subsets $P^{*}(X)$ of a power set $P(X)$ of $X$ as follows:

1. If $|X| \geq 5$ then $P^{*}(X):=P(X) \backslash(\{\emptyset, X\} \cup\{\{x\}: x \in$ $X\})$,

2. if $|X|=4$ then $P^{*}(X):=\{Y \subseteq X:|Y| \geq 2$, $|X \backslash Y|=2$ or $\left.\left\{x_{2}, x_{3}\right\} \subseteq Y\right\}$ and

3. if $|X|=3$ then $P^{*}(X):=\left\{Y \subseteq X:|Y|=2, x_{2} \in Y\right\}$.

Then we obtain the results as shown in the following theorems.

Theorem 2.3. [16] If $Y \in P^{*}(X)$ then $\operatorname{rank}(\mathcal{T}(X, Y)$ : $\mathcal{O}(X, Y))=S(n, m)-\left(\begin{array}{c}n-1 \\ m-1\end{array}\right)$.

Theorem 2.4. [16] If $Y \notin P^{*}(X)$ then $\operatorname{rank}(\mathcal{T}(X, Y)$ : $\mathcal{O}(X, Y))=S(n, m)-\left(\begin{array}{c}n-1 \\ m-1\end{array}\right)+1$.

Lemma 2.5. Let $B \subseteq \mathcal{T}(X, Y) \backslash \mathcal{O D}(X, Y)$ and $\langle\mathcal{O D}(X, Y), B\rangle=\mathcal{T}(X, Y)$. Then $\mathcal{K} \subseteq\{\operatorname{ker} \phi: \phi \in B\}$.

Proof. Suppose that $B \subseteq \mathcal{T}(X, Y) \backslash \mathcal{O D}(X, Y)$ with $\langle\mathcal{O D}(X, Y), B\rangle=\mathcal{T}(X, Y)$. Assume that there exists $K \in \mathcal{K}$ with $K \notin\{\operatorname{ker} \phi: \phi \in B\}$. Since $\alpha_{K} \in \mathcal{T}(X, Y)=$ $\langle\mathcal{O D}(X, Y), B\rangle$, there are $\theta_{1} \in \mathcal{O} \mathcal{D}(X, Y) \cup B$ and $\theta_{2} \in$ 
$\mathcal{T}(X, Y)$ such that $\alpha_{K}=\theta_{1} \theta_{2}$. Because $\operatorname{rank}_{K}=m$, we obtain $\operatorname{ker} \alpha_{K}=\operatorname{ker} \theta_{1}$, i.e. $\operatorname{ker} \theta_{1}=K$. Hence, $\theta_{1} \notin B$ and $\theta_{1} \notin \mathcal{O D}(X, Y)$ because $K \notin\{\operatorname{ker} \phi: \phi \in \mathcal{O D}(X, Y)\}$ which is a contradiction.

Theorem 2.6. Let $\mathcal{S} \subseteq \mathcal{S}(Y)$ and $\langle\mathcal{S}\rangle=\mathcal{S}(Y)$. Then

$$
\mathcal{T}(X, Y)=\left\langle\mathcal{O D}(X, Y),\left\{\mu_{s}: s \in \mathcal{S}\right\},\left\{\alpha_{K}: K \in \mathcal{K}\right\}\right\rangle .
$$

Proof. By Lemma 2.2 and $\mathcal{O}(X, Y)$ is a proper subsemigroup of $\mathcal{O D}(X, Y), \mathcal{T}(X, Y)=\left\langle\mathcal{O}(X, Y),\left\{\mu_{s}: s \in \mathcal{S}\right\},\left\{\alpha_{K}:\right.\right.$ $K \in \mathcal{K}\}\rangle \subseteq\left\langle\mathcal{O D}(X, Y),\left\{\mu_{s}: s \in \mathcal{S}\right\},\left\{\alpha_{K}: K \in \mathcal{K}\right\}\right\rangle$. It is clear that $\left\langle\mathcal{O D}(X, Y),\left\{\mu_{s}: s \in \mathcal{S}\right\},\left\{\alpha_{K}: K \in\right.\right.$ $\mathcal{K}\}\rangle \subseteq \mathcal{T}(X, Y)$. Altogether, we obtain that $\mathcal{T}(X, Y)=$ $\left\langle\mathcal{O D}(X, Y),\left\{\mu_{s}: s \in \mathcal{S}\right\},\left\{\alpha_{K}: K \in \mathcal{K}\right\}\right\rangle$.

From Theorem 2.6, we obtain the following corollaries.

Corollary 2.7. If $|X| \geq 5$ and $|Y| \geq 3$ then $\operatorname{rank}(\mathcal{T}(X, Y)$ : $\mathcal{O D}(X, Y))=S(n, m)-\left(\begin{array}{c}n-1 \\ m-1\end{array}\right)$.

Proof. Since $|Y| \geq 3$, two bijections, say $s_{1}$ and $s_{2}$, generate the symmetric group $\mathcal{S}(Y)$. Then there are $K_{1}, K_{2} \notin$ $\{\operatorname{ker} \phi: \phi \in \mathcal{O D}(X, Y)\}$, i.e. $K_{1}, K_{2} \in \mathcal{K}$ and $Y$ is a transversal of $K_{1}, K_{2}$. Without loss of generality, we can assume that $\left.\alpha_{K_{1}}\right|_{Y}=s_{1}$ and $\left.\alpha_{K_{2}}\right|_{Y}=s_{2}$, i.e. $\mu_{s_{1}}=\alpha_{K_{1}}$ and $\mu_{s_{2}}=\alpha_{K_{2}}$. By Theorem 2.6, we have $\mathcal{T}(X, Y)=$ $\left\langle\mathcal{O D}(X, Y),\left\{\alpha_{K}: K \in \mathcal{K}\right\}\right\rangle$. Hence, $\operatorname{rank}(\mathcal{T}(X, Y) \quad:$ $\mathcal{O D}(X, Y)) \leq\left|\left\{\alpha_{K}: K \in \mathcal{K}\right\}\right|=S(n, m)-\left(\begin{array}{c}n-1 \\ m-1\end{array}\right)$. Вy Lemma 2.5, we obtain that $\operatorname{rank}(\mathcal{T}(X, Y): \mathcal{O D}(X, Y)) \geq$ $|\mathcal{K}|=S(n, m)-\left(\begin{array}{c}n-1 \\ m-1\end{array}\right)$.

Corollary 2.8. If $|X| \geq 3$ and $|Y|=2$ then $\operatorname{rank}(\mathcal{T}(X, Y)$ : $\mathcal{O D}(X, Y))=2^{n-1}-n$.

Proof. Since $|Y|=2$, one bijection, say $s_{1}$, generates the symmetric group $\mathcal{S}(Y)$. Then there is $K_{1} \in\{\operatorname{ker} \beta$ : $\beta \in \mathcal{O D}(X, Y)\}$ such that $Y$ is a transversal of $K_{1}$. Without loss of generality, we can assume that $\left.\alpha_{K_{1}}\right|_{Y}=s_{1}$, i.e. $\mu_{s_{1}}=\alpha_{K_{1}}$. By Theorem 2.6, we have $\mathcal{T}(X, Y)=$ $\left\langle\mathcal{O D}(X, Y),\left\{\alpha_{K}: K \in \mathcal{K}\right\}\right\rangle$. Then we have $\operatorname{rank}(\mathcal{T}(X, Y)$ : $\mathcal{O D}(X, Y)) \leq\left|\left\{\alpha_{K}: K \in \mathcal{K}\right\}\right|=S(n, 2)-\left(\begin{array}{l}n-1 \\ 2-1\end{array}\right)=$ $2^{n-1}-n$. By Lemma 2.5, we obtain $\operatorname{rank}(\mathcal{T}(X, Y)$ : $\mathcal{O D}(X, Y)) \geq|\mathcal{K}|=S(n, 2)-\left(\begin{array}{l}n-1 \\ 2-1\end{array}\right)=2^{n-1}-n$.

Corollary 2.9. Let $|X|=4$ and $|Y|=3$. If $\left\{x_{2}, x_{3}\right\} \nsubseteq Y$ and $\left\{x_{2}, x_{3}\right\} \subseteq Y$ then $\operatorname{rank}(\mathcal{T}(X, Y): \mathcal{O D}(X, Y))=4$ and $\operatorname{rank}(\mathcal{T}(X, Y): \mathcal{O D}(X, Y))=3$, respectively.

Proof. Since $|Y|=3$, two bijections, say $s_{1}$ and $s_{2}$, generate the symmetric group $\mathcal{S}(Y)$. If $\left\{x_{2}, x_{3}\right\} \nsubseteq Y$ then there is only one $K_{1} \in \mathcal{K}$ such that $Y$ is a transversal of $K_{1}$ and $K_{1} \notin\{\operatorname{ker} \phi: \phi \in \mathcal{O D}(X, Y)\}$. Without loss of generality, we can assume that $\left.\alpha_{K_{1}}\right|_{Y}=s_{1}$, i.e. $\mu_{s_{1}}=\alpha_{K_{1}}$. Then $\mu_{s_{2}} \notin \mathcal{O D}(X, Y) \cup\left\{\alpha_{K}: K \in\right.$ $\mathcal{K}\}$. Put $\alpha:=\mu_{s_{2}}$ and $\alpha_{K_{1}}:=\mu_{s_{1}}$. By Theorem
2.6, we have $\mathcal{T}(X, Y)=\left\langle\mathcal{O D}(X, Y),\left\{\alpha_{K}: K \in \mathcal{K}\right\}, \alpha\right\rangle$. So, we obtain that $\operatorname{rank}(\mathcal{T}(X, Y): \mathcal{O D}(X, Y)) \leq$ $\left|\left\{\alpha_{K}: K \in \mathcal{K}\right\} \cup\{\alpha\}\right|=S(4,3)-\left(\begin{array}{l}4-1 \\ 3-1\end{array}\right)+1=4$. Since there exists only one $K_{1} \in \mathcal{K}$ such that $Y$ is a transversal of $K_{1}$ and $K_{1} \notin\{\operatorname{ker} \phi: \phi \in \mathcal{O D}(X, Y)\}$. Thus, we want one element in $B$ which is not in $\mathcal{O D}(X, Y) \cup\left\{\alpha_{K}: K \in \mathcal{K}\right\}$. Hence, $|B| \geq|\mathcal{K}|+1=S(4,3)-\left(\begin{array}{l}4-1 \\ 3-1\end{array}\right)+1=4$.

For the case $\left\{x_{2}, x_{3}\right\} \subseteq Y$, the proof is similar to Corollary 2.7. Then we have $\operatorname{rank}(\mathcal{T}(X, Y): \mathcal{O D}(X, Y))=S(4,3)-$ $\left(\begin{array}{l}4-1 \\ 3-1\end{array}\right)=3$

Example 2.10. Let $X=\{1,2,3\}$ and $Y=\{1,2\}$. Then $\operatorname{rank}(\mathcal{T}(X, Y): \mathcal{O D}(X, Y))=1$.

Solution. So, we have $|X|=3,|Y|=2$, and $|\mathcal{K}|=1$. Put $\theta:=\left(\begin{array}{cc}1 & 2,3 \\ 2 & 1\end{array}\right)$ and $\alpha:=\left(\begin{array}{cc}1,3 & 2 \\ 1 & 2\end{array}\right)$. It is clear that $\theta \in \mathcal{O D}(X, Y), \operatorname{ker} \theta \in\{\operatorname{ker} \phi: \phi \in \mathcal{O} \mathcal{D}(X, Y)\}$ with $Y$ is a transversal of $\operatorname{ker} \theta$, and $\left\langle\left.\theta\right|_{Y}\right\rangle=\mathcal{S}(Y)$. By the definition of $\alpha$, we have $\operatorname{ker} \alpha \in \mathcal{K}$. Since $|\mathcal{K}|=1$, there is exactly one $K \in \mathcal{K}$. Without loss of generality, we can assume that $\alpha_{K}=\alpha$. So, it is easy to see that $\alpha_{K} \in \mathcal{T}(X, Y) \backslash \mathcal{O D}(X, Y)$ and $\left\langle\mathcal{O D}(X, Y), \alpha_{K}\right\rangle=\mathcal{T}(X, Y)$. Hence, $\operatorname{rank}(\mathcal{T}(X, Y)$ : $\mathcal{O D}(X, Y))=1$ which satisfies a result in Corollary 2.8.

\section{$2.2 \operatorname{Rank}(\mathcal{T}(X, Y): \mathcal{O P}(X, Y))$}

In this section, we study and describe the relative rank of $\mathcal{T}(X, Y)$ modulo $\mathcal{O P}(X, Y)$. Assume that $Y$ is a proper subset of $X$. Define the set $\mathcal{P}$ by

$$
\begin{gathered}
\mathcal{P}:=\{\operatorname{ker} \alpha: \alpha \in \mathcal{O P}(X, Y), \operatorname{rank} \alpha=m\} \backslash\{\operatorname{ker} \alpha: \alpha \in \\
\mathcal{O}(X, Y), \operatorname{rank} \alpha=m\} .
\end{gathered}
$$

Then $\mathcal{P}$ is the set of all partitions of $X$ into $m-1$ intervals and one block which is the union of two intervals $B_{1}$ and $B_{n}$ such that $1 \in B_{1}$ and $n \in B_{n}$. For each $P \in \mathcal{P}$, we fix any $\alpha_{P} \in \mathcal{O P}(X, Y)$ with $i m \alpha_{P}=Y$ and $\operatorname{ker} \alpha_{P}=P$. The following lemma gives the cardinality of $\mathcal{P}$.

Lemma 2.11. $[4]|\mathcal{P}|=\left|\left\{\alpha_{P}: P \in \mathcal{P}\right\}\right|=\left(\begin{array}{c}n-1 \\ m\end{array}\right)$.

Next, we define a mapping $\alpha^{*}: X \rightarrow Y$ by

$$
x \alpha^{*}:= \begin{cases}l_{i+1} & \text { if } l_{i} \leq x<l_{i+1} \text { and } 1 \leq i<m \\ l_{1} & \text { if } l_{m} \leq x \text { or } x<l_{1} .\end{cases}
$$

Then $\left.\alpha^{*}\right|_{Y}$ is a permutation on $Y$ and $\left(l_{1} \alpha^{*}, \ldots, l_{m} \alpha^{*}\right)$ is cyclic, i.e. $\alpha^{*} \in \mathcal{O P}(X, Y)$. Since $\mathcal{O}(X, Y) \subseteq \mathcal{O P}(X, Y)$, we have $\operatorname{rank}(\mathcal{T}(X, Y): \mathcal{O P}(X, Y)) \leq \operatorname{rank}(\mathcal{T}(X, Y)$ : $\mathcal{O}(X, Y))$, i.e. $\operatorname{rank}(\mathcal{T}(X, Y): \mathcal{O P}(X, Y)) \leq S(n, m)-$ $\left(\begin{array}{c}n-1 \\ m-1\end{array}\right)+1$. Define the set $\mathcal{M}$ by

$$
\begin{gathered}
\mathcal{M}:=\{\operatorname{ker} \alpha: \alpha \in \mathcal{T}(X, Y), \operatorname{rank} \alpha=m\} \backslash\{\operatorname{ker} \alpha: \alpha \in \\
\mathcal{O P}(X, Y), \operatorname{rank} \alpha=m\} .
\end{gathered}
$$

Then the following lemma gives the cardinality of $\mathcal{M}$. 
Lemma 2.12. $[3]|\mathcal{M}|=S(n, m)-\left(\begin{array}{c}n \\ m\end{array}\right)$.

Notice that for any $\beta \in \mathcal{S}(Y)$, there is $\beta^{\prime} \in \mathcal{T}(X, Y)$ such that $\left.\beta^{\prime}\right|_{Y}=\beta$, i.e. $\mathcal{S}(Y) \subseteq\left\{\left.\alpha\right|_{Y}: \alpha \in \mathcal{T}(X, Y)\right\}$. The following lemmas give necessary and sufficient conditions for a set $A \subseteq \mathcal{T}(X, Y)$ to be a generating set of $\mathcal{T}(X, Y)$ modulo $\mathcal{O P}(X, Y)$

Lemma 2.13. [3] For any generating set $A$ of $\mathcal{T}(X, Y)$ modulo $\mathcal{O P}(X, Y)$, there is a set $C \subseteq A$ with $\mathcal{M} \subseteq\{\operatorname{ker} \alpha: \alpha \in$ $C\}$ such that there is a set $B \subseteq C$ with $\left\langle\left\{\left.\alpha\right|_{Y}: \alpha \in B\right\}, \eta\right\rangle=$ $\mathcal{S}(Y)$ where $\eta=\left.\alpha^{*}\right|_{Y}$.

Lemma 2.14. [3] Let $A \subseteq \mathcal{T}(X, Y) \backslash \mathcal{O P}(X, Y)$ with $\mathcal{M} \subseteq$ $\{\operatorname{ker} \alpha: \alpha \in A\}$ and $\mathcal{S}(Y)=\left\langle\left\{\left.\alpha\right|_{Y}: \alpha \in B\right\}, \eta\right\rangle$ for some $B \subseteq A$. Then $A$ is a generating set of $\mathcal{T}(X, Y)$ modulo $\mathcal{O P}(X, Y)$.

Then we obtain the main result of this section as shown in the following theorem.

Theorem 2.15. [3] $\operatorname{rank}(\mathcal{T}(X, Y): \mathcal{O P}(X, Y))=|\mathcal{M}|$.

Proof. By Lemma 2.13, we get that $\operatorname{rank}(\mathcal{T}(X, Y)$ : $\mathcal{O P}(X, Y)) \geq|\mathcal{M}|$. For all $M \in \mathcal{M}$, we fix $\alpha_{M} \in$ $\mathcal{T}(X, Y) \backslash \mathcal{O P}(X, Y)$ with $\operatorname{ker} \alpha_{M}=M$. Without loss of generality, we can assume that there is $\beta \in\left\{\alpha_{M}: M \in \mathcal{M}\right\}$ with $\left.\beta\right|_{Y}:=\left(\begin{array}{lllll}l_{1} & l_{2} & l_{3} & \cdots & l_{m} \\ l_{2} & l_{1} & l_{3} & \cdots & l_{m}\end{array}\right)$. It is well known that $\left\langle\left.\beta\right|_{Y}, \eta\right\rangle=\mathcal{S}(Y)$ where $\eta=\left.\alpha^{*}\right|_{Y}$. By Lemma 2.14, we have $\left\langle\mathcal{O P}(X, Y),\left\{\alpha_{M}: M \in \mathcal{M}\right\}\right\rangle=\mathcal{T}(X, Y)$. Then $\operatorname{rank}(\mathcal{T}(X, Y): \mathcal{O P}(X, Y)) \leq\left|\left\{\alpha_{M}: M \in \mathcal{M}\right\}\right|=|\mathcal{M}|$. Altogether, $\operatorname{rank}(\mathcal{T}(X, Y): \mathcal{O P}(X, Y))=|\mathcal{M}|$.

Example 2.16. Let $X=\{1,2,3,4\}$ and $Y=\{1,2,3\}$. Then $\operatorname{rank}(\mathcal{T}(X, Y): \mathcal{O P}(X, Y))=2$.

Solution. So, we have $|X|=4,|Y|=3$, and $|\mathcal{M}|=2$. Then we put $\alpha^{*}:=\left(\begin{array}{ccc}1 & 2 & \overline{3,4} \\ 2 & 3 & 1\end{array}\right), \beta:=\left(\begin{array}{ccc}1 & \overline{2,4} & 3 \\ 2 & 1 & 3\end{array}\right)$, and $\gamma:=\left(\begin{array}{ccc}\overline{1,3} & 2 & 4 \\ 1 & 2 & 3\end{array}\right)$. It is immediately to see that $\alpha^{*} \in$ $\mathcal{O P}(X, Y)$. By the definition of $\beta$ and $\gamma$, we obtain that $\operatorname{ker} \beta$, ker $\gamma \in \mathcal{M}$ and $\operatorname{ker} \beta \neq \operatorname{ker} \gamma$. Since $|\mathcal{M}|=2$, there are only $M_{1}, M_{2} \in \mathcal{M}$. Without loss of generality, we can assume that $\alpha_{M_{1}}=\beta$ and $\alpha_{M_{2}}=\gamma$. Since $Y$ is a transversal of $\operatorname{ker} \alpha^{*}$ and ker $\alpha_{M_{1}}$, we can show that $\left\langle\left.\alpha^{*}\right|_{Y},\left.\alpha_{M_{1}}\right|_{Y}\right\rangle=\mathcal{S}(Y)$. Then it is easy to verify that $\left\langle\mathcal{O P}(X, Y), \alpha_{M_{1}}, \alpha_{M_{2}}\right\rangle=\mathcal{T}(X, Y)$. So, $\operatorname{rank}(\mathcal{T}(X, Y): \mathcal{O P}(X, Y))=2$ which satisfies a result in Theorem 2.15.

\section{$2.3 \operatorname{Rank}(\mathcal{T}(X, Y): \mathcal{O P} \mathcal{R}(X, Y))$}

The both semigroups $\mathcal{O P R}(X, Y)$ and $\mathcal{O P}(X, Y)$ coinside when $Y$ has only two elements. So, we will consider $Y$ is a proper subset of $X$ with $|Y| \geq 3$. Recall that $\mathcal{O P R}(X, Y)=\mathcal{O P}(X, Y) \cup \mathcal{O R}(X, Y)$ and notice that $\{\operatorname{ker} \alpha: \alpha \in \mathcal{O P}(X, Y)\}=\{\operatorname{ker} \alpha: \alpha \in \mathcal{O P} \mathcal{P}(X, Y)\}$. Define a mapping $\beta^{*}: X \rightarrow Y$ by

$$
x \beta^{*}:= \begin{cases}l_{m} & \text { if } x<l_{1} \\ l_{m-i+1} & \text { if } l_{i} \leq x<l_{i+1} \text { and } 1 \leq i<m \\ l_{1} & \text { if } x \geq l_{m} .\end{cases}
$$

We note that $\beta^{*}$ is orientation-reversing. Then we obtain that $\mathcal{O P}(X, Y)$ and $\beta^{*}$ will be a generating set for $\mathcal{O P R}(X, Y)$ as shown in the following theorem.

Theorem 2.17. $\mathcal{O P} \mathcal{R}(X, Y)=\left\langle\mathcal{O P}(X, Y), \beta^{*}\right\rangle$.

Proof. Let $\gamma \in \mathcal{O} \mathcal{R}(X, Y)$ with $\operatorname{rank} \gamma=m$. We put $\theta:=\gamma \beta^{*}$ and observe that $\theta \in \mathcal{O P}(X, Y)$ as a product of two orientation-reversing transformations. Then $\theta \beta^{*}=$ $\gamma \beta^{*} \beta^{*}=\gamma$ since $\left.\beta^{*} \beta^{*}\right|_{Y}$ is the identity mapping on $Y$, i.e. $\gamma \in\left\langle\mathcal{O P}(X, Y), \beta^{*}\right\rangle$.

Let $2 \leq p \in \mathbb{N}$ with $p \leq m$ and suppose that $\{\alpha \in$ $\mathcal{O} \mathcal{R}(X, Y): \operatorname{rank} \alpha=p\} \subseteq\left\langle\mathcal{O P}(X, Y), \beta^{*}\right\rangle$. Let $\gamma \in$ $\mathcal{O R}(X, Y)$ with $\operatorname{rank} \gamma=p-1$. We observe that $\operatorname{ker} \gamma=$ $\left\{G_{1}, G_{2}<\cdots<G_{p-1}\right\}$, where $G_{1}<G_{2}$ or $\{1, n\} \subseteq G_{1}$, where $i m \gamma=\left\{k_{1}, \ldots, k_{p-1}\right\}$ with $k_{i} \gamma^{-1}=G_{i}$ for $1 \leq i \leq$ $p-1$. Let us put $k_{p}:=k_{1}$ due to technical reasons. Since $\gamma$ is orientation-reversing, there is one $r \in\{1, \cdots, p-1\}$ such that $k_{r}<k_{r+1}$ and $k_{i}>k_{i+1}$ for all $i \in\{1, \cdots, p-1\} \backslash\{r\}$. Since $p-1<m$, we have $Y \backslash i m \gamma \neq \emptyset$. So, there are $b \in Y \backslash i m \gamma$ and $i \in\{1, \ldots, p-1\}$ such that (i) $k_{i}>b>k_{i+1}$ or (ii) $k_{i}<b$ and $b>k_{i+1}$ or (iii) $k_{i}>b$ and $b<k_{i+1}$. In all the three cases, we put

$$
m_{j}:= \begin{cases}k_{j} & \text { if } 1 \leq j \leq i \\ b & \text { if } j=i+1 \\ k_{j-1} & \text { if } i+1<j \leq p\end{cases}
$$

Let $q \in\{1, \ldots, p\}$ such that $m_{q}$ is the least element in $B:=$ $\left\{m_{1}, \ldots, m_{p}\right\}$. Next, we define a mapping $\delta: B \rightarrow B$ by

$$
m_{j} \delta:= \begin{cases}m_{q+1-j} & \text { if } 1 \leq j \leq q \\ m_{p+q+1-j} & \text { if } q+1 \leq j \leq p .\end{cases}
$$

Clearly, $\delta$ is bijective. Further, we define a mapping $\delta^{*}: X \rightarrow$ $Y$ by

$x \delta^{*}:= \begin{cases}m_{1} & \text { if } x<m_{1} \delta^{-1} \\ m_{j} & \text { if } m_{j} \delta^{-1} \leq x<m_{j+1} \delta^{-1} \text { and } 1 \leq j \leq p-1 \\ m_{p} & \text { if } x \geq m_{p} \delta^{-1}\end{cases}$

It is easy to verify that $m_{1}\left(\delta^{*}\right)^{-1}<\cdots<m_{p}\left(\delta^{*}\right)^{-1}$, $m_{q}<m_{q+1}$, and $m_{j}>m_{j+1}$ for all $j \in\{1, \ldots, p\} \backslash\{q\}$, where $m_{p+1}:=m_{1}$. Thus, $\delta^{*}$ is an orientation-reversing transformation with $\operatorname{rank} \delta^{*}=p$, i.e. $\delta^{*} \in\left\langle\mathcal{O P}(X, Y), \beta^{*}\right\rangle$ by the assumption. Moreover, we observe that $\delta=\left.\delta^{*}\right|_{B}$. Let $\theta: X \rightarrow B$ be defined by

$$
x \theta:= \begin{cases}m_{j} \delta^{-1} & \text { if } x \in G_{j} \text { and } 1 \leq j \leq i \\ m_{j+1} \delta^{-1} & \text { if } x \in G_{j} \text { and } i<j \leq p-1 .\end{cases}
$$

Notice that $\operatorname{ker} \theta=\operatorname{ker} \gamma$. Since $m_{j} \delta^{-1}<m_{j+1} \delta^{-1}$ for all $j \in\{1, \ldots, p-1\}$, it is easy to verify that $\theta \in \mathcal{O}(X, Y) \subseteq$ $\mathcal{O P}(X, Y)$.

Finally, we show that $\gamma=\theta \delta^{*}$. Let $x \in X$. Then $x \in G_{j}$ for some $j \in\{1, \ldots, p-1\}$. If $1 \leq j \leq i$ then we get $x \theta \delta^{*}=x \theta \delta=m_{j} \delta^{-1} \delta=m_{j}=k_{j}=x \gamma$. If $i<j \leq p-1$ then we have $x \theta \delta^{*}=x \theta \delta=m_{j+1} \delta^{-1} \delta=m_{j+1}=k_{j}=x \gamma$. Hence, we have $\gamma=\theta \delta^{*} \in\left\langle\mathcal{O P}(X, Y), \beta^{*}\right\rangle$.

From Theorem 2.17, we obtain immediately the following corollary. 
Corollary 2.18. $\operatorname{rank}(\mathcal{O P} \mathcal{R}(X, Y): \mathcal{O P}(X, Y))=1$.

Proof. By Theorem 2.17, we obtain that $\operatorname{rank}(\mathcal{O P R}(X, Y)$ : $\mathcal{O P}(X, Y)) \leq 1$. Since $\mathcal{O P}(X, Y)$ is a proper subsemigroup of $\mathcal{O P R}(X, Y)$, we obtain that $\operatorname{rank}(\mathcal{O P R}(X, Y)$ : $\mathcal{O P}(X, Y)) \geq 1$. Altogether, we can conclude that $\operatorname{rank}(\mathcal{O P R}(X, Y): \mathcal{O P}(X, Y))=1$.

Example 2.19. Let $X=\{1,2,3,4\}$ and $Y=\{2,3,4\}$. Then $\operatorname{rank}(\mathcal{O P R}(X, Y): \mathcal{O P}(X, Y))=1$.

Solution. So, we have $|X|=4$ and $|Y|=3$. Then we put $\beta^{*}:=\left(\begin{array}{ccc}\overline{1,2} & 3 & 4 \\ 4 & 3 & 2\end{array}\right)$. By the definition of $\beta^{*}$, we obtain that $\beta^{*}$ is orientation-reversing, i.e. $\beta^{*} \in \mathcal{O} \mathcal{R}(X, Y)$. Hence, it is easy to show that $\left\langle\mathcal{O P}(X, Y), \beta^{*}\right\rangle=\mathcal{O P R}(X, Y)$, i.e. $\operatorname{rank}(\mathcal{O P} \mathcal{R}(X, Y): \mathcal{O P}(X, Y))=1$ which satisfies a result in Corollary 2.18.

Lemma 2.20. For any generating set $A$ of $\mathcal{T}(X, Y)$ modulo $\mathcal{O P R}(X, Y)$, there is a set $A^{\prime} \subseteq A$ with $\mathcal{M}=\{\operatorname{ker} \alpha: \alpha \in$ $\left.A^{\prime}\right\}$.

Proof. Suppose that $A \subseteq \mathcal{T}(X, Y) \backslash \mathcal{O P R}(X, Y)$ with $\langle\mathcal{O P R}(X, Y), A\rangle=\mathcal{T}(X, Y)$. Assume that there is $M \in \mathcal{M}$ with $M \notin\{\operatorname{ker} \alpha: \alpha \in A\}$. Let $\gamma$ be a transformation with $\operatorname{ker} \gamma=M$, i.e. $\operatorname{rank} \gamma=m$. Since $\gamma \in \mathcal{T}(X, Y)=$ $\langle\mathcal{O P R}(X, Y), A\rangle$, there are $\theta_{1} \in \mathcal{O P R}(X, Y) \cup A$ and $\theta_{2} \in \mathcal{T}(X, Y)$ such that $\gamma=\theta_{1} \theta_{2}$. Because $\operatorname{rank} \gamma=m$, we obtain that $\operatorname{ker} \gamma=\operatorname{ker} \theta_{1}$, i.e. $\operatorname{ker} \theta_{1}=M$. We have $\operatorname{ker} \theta_{1}=M \notin\{\operatorname{ker} \alpha: \alpha \in \mathcal{O P}(X, Y)\}$. Moreover, it is clear that $\{\operatorname{ker} \alpha: \alpha \in \mathcal{O P R}(X, Y)\}=\{\operatorname{ker} \alpha: \alpha \in$ $\mathcal{O P}(X, Y)\}$. Thus, $\operatorname{ker} \theta_{1} \notin\{\operatorname{ker} \alpha: \alpha \in \mathcal{O P R}(X, Y)\}$, i.e. $\theta_{1} \notin \mathcal{O P R}(X, Y)$. Additional, it holds that $\theta_{1} \notin A$ since $\mathcal{M} \notin\{\operatorname{ker} \alpha: \alpha \in A\}$, i.e. $\theta_{1} \notin \mathcal{O P R}(X, Y) \cup A$, a contradiction. This shows that there is a set $A^{\prime} \subseteq A$ with $\left\{\operatorname{ker} \alpha: \alpha \in A^{\prime}\right\}=\mathcal{M}$.

Then we can state the main result as follows:

Theorem 2.21. $\operatorname{rank}(\mathcal{T}(X, Y): \quad \mathcal{O P R}(X, Y))=$ $\operatorname{rank}(\mathcal{T}(X, Y): \mathcal{O P}(X, Y))$.

Proof. Since $\mathcal{O P}(X, Y) \subseteq \mathcal{O P R}(X, Y)$, we obtain that $\operatorname{rank}(\mathcal{T}(X, Y) \quad: \quad \mathcal{O P} \mathcal{R}(X, Y)) \leq \operatorname{rank}(\mathcal{T}(X, Y):$ $\mathcal{O P}(X, Y))=|\mathcal{M}|$ by Theorem 2.15. Conversely, let $A \subseteq \mathcal{T}(X, Y) \backslash \mathcal{O P R}(X, Y)$ such that $\langle\mathcal{O P} \mathcal{R}(X, Y), A\rangle=$ $\mathcal{T}(X, Y)$. By Lemma 2.20, there is $A^{\prime} \subseteq A$ with $\mathcal{M}=$ $\left\{\operatorname{ker} \alpha: \alpha \in A^{\prime}\right\}$, i.e. $\operatorname{rank}(\mathcal{T}(X, Y): \mathcal{O P} \mathcal{R}(X, Y)) \geq$ $\left|A^{\prime}\right| \geq|\mathcal{M}|$. Therefore, $\operatorname{rank}(\mathcal{T}(X, Y): \mathcal{O P} \mathcal{R}(X, Y))=$ $|\mathcal{M}|=\operatorname{rank}(\mathcal{T}(X, Y): \mathcal{O P}(X, Y))$.

Example 2.22. Let $X=\{1,2,3,4\}$ and $Y=\{1,2,3\}$. Then $\operatorname{rank}(\mathcal{T}(X, Y): \mathcal{O P R}(X, Y))=2$.

Solution. So, we have $|X|=4,|Y|=3$, and $|\mathcal{M}|=2$. Then we put $\alpha^{*}:=\left(\begin{array}{ccc}1 & 2 & \overline{3,4} \\ 2 & 3 & 1\end{array}\right), \beta:=\left(\begin{array}{ccc}1 & \overline{2,4} & 3 \\ 2 & 1 & 3\end{array}\right)$, and $\gamma:=\left(\begin{array}{ccc}\overline{1,3} & 2 & 4 \\ 1 & 2 & 3\end{array}\right) . \quad$ It is clear that $\alpha^{*} \in \mathcal{O P}(X, Y) \subseteq$
$\mathcal{O P R}(X, Y)$. By the definition of $\beta$ and $\gamma$, we obtain that $\operatorname{ker} \beta, \operatorname{ker} \gamma \in \mathcal{M}$ and $\operatorname{ker} \beta \neq \operatorname{ker} \gamma$. Since $|\mathcal{M}|=2$, there are only $M_{1}, M_{2} \in \mathcal{M}$. Without loss of generality, we can assume that $\alpha_{M_{1}}=\beta$ and $\alpha_{M_{2}}=\gamma$. Then we have $\alpha_{M_{1}}, \alpha_{M_{2}} \in$ $\mathcal{T}(X, Y) \backslash \mathcal{O P R}(X, Y)$. Since $Y$ is a transversal of $\operatorname{ker} \alpha^{*}$ and $\operatorname{ker} \alpha_{M_{1}}$, we can show that $\left\langle\left.\alpha^{*}\right|_{Y},\left.\alpha_{M_{1}}\right|_{Y}\right\rangle=\mathcal{S}(Y)$. Hence, it is easy to calculate that $\left\langle\mathcal{O P R}(X, Y), \alpha_{M_{1}}, \alpha_{M_{2}}\right\rangle=$ $\mathcal{T}(X, Y)$. Then $\operatorname{rank}(\mathcal{T}(X, Y): \mathcal{O P R}(X, Y))=2$ which satisfies a result in Theorem 2.21.

\section{Conclusions}

In this paper, we study transformation semigroup $\mathcal{T}(X)$ and transformation semigroup with restricted range $\mathcal{T}(X, Y)$. We also determine the relative rank of transformation semigroup $\mathcal{T}(X, Y)$ modulo various subsemigroups in $\mathcal{T}(X, Y)$. In Section 1 , we define some notation and introduce some definition about transformation semigroups in order to use through this paper. In section 2.1, we obtain the relative rank of $\mathcal{T}(X, Y)$ modulo $\mathcal{O D}(X, Y)$ as shown in Theorem 2.6 and Corollary 2.7-2.9. In section 2.2 , we study and describe the relative rank of $\mathcal{T}(X, Y)$ modulo $\mathcal{O P}(X, Y)$ as shown in Theorem 2.15. In section 2.3, we calculate the relative rank of $\mathcal{O P R}(X, Y)$ modulo $\mathcal{O P}(X, Y)$ as shown in Theorem 2.17 and Corollary 2.18. In addition, we obtain that the relative rank of $\mathcal{T}(X, Y)$ modulo $\mathcal{O P}(X, Y)$ and modulo $\mathcal{O P} \mathcal{R}(X, Y)$ coincide as shown in Theorem 2.21. Finally, we also illustrate examples in each section in order to get a good understandable.

In future work, we can study other kind structure of transformation semigroup with restricted range.

\section{Acknowledgements}

This work was financially supported by Prince of Songkla University, Pattani Campus, Pattani 94000, Thailand.

\section{REFERENCES}

[1] R.E. Arthur and N. Ruškuc, Presentations for two extensions of the monoid of order-preserving mappings on a finite chain, Southeast Asian Bulletin of Mathematics 24,1-7, 2000.

[2] P.M. Cartarino and P.M. Higgins, The monoid of orientationpreserving mappings on a chain, Semigroup Forum 58,190-206, 1999.

[3] I. Dimitrova and J. Koppitz, On relative ranks of finite transformation semigroups with restricted range, arXiv:2006.07724 [math.RA], 2020.

[4] I. Dimitrova, J. Koppitz, and K. Tinpun, On the relative rank of the semigroup of orientation-preserving transformations with restricted range, Proceeding of 47th Spring Conference of Union of Bulgarian Mathematics Borovets , April 2-6, 109-114, 2018. 
[5] I. Dimitrova, V.H. Fernandes, and J. Koppitz, The maximal subsemigroups of semigroups of transformations preserving or reversing the orientation on a finite chain, Publ. Math. Debrecen. 81, no.1-2, 11-29, 2012.

[6] G.M.S. Gomes and J.M. Howie, On the ranks of certain semigroups of order-preserving transformations, Semigroup Forum 45, 272-282, 1992.

[7] V.H. Fernandes, G.M.S. Gomes, and M.M. Jesus, Congruences on monoids of transformations preserving the orientation on a finite chain, Journal of Algebra 321, 743-757, 2009.

[8] V.H. Fernandes, P. Honyam, T.M. Quinteiro, and B. Singha, On semigroups of endomorphisms of a chain with restriced range, Semigroup Forum 89, 77-104, 2013.

[9] V.H. Fernandes, P. Honyam, T.M. Quinteiro, and B. Singha, On semigroups of orientation-preserving transformations with restricted range, Communications in Algebra 44, 253-264, 2016.

[10] V.H. Fernandes and T.M. Quinteiro, The cardinal of various monoids of transformations that preserve a uniform partition, Bull. Malays. Math. Sci. Soc. 35, no. 4, 885-896, 2012.

[11] V.H. Fernandes and T.M. Quinteiro, On the ranks of certain monoids of transformations that preserve a uniform partition, Communications in Algebra 42, 615-636, 2014.
[12] V. H. Fernandes and J. Sanwong, On the ranks of semigroups of transformations on a finite set with restricted range, Algebra Collog. 21, no. 3, 497-510, 2014.

[13] J.M. Howie, N. Ruškuc, and P.M. Higgins, On relative ranks of full transformation semigroups, Communications in Algebra 26, no. 3, 733-748, 1998.

[14] D.B. McAlister, Semigroups generated by a group and an idempotent, Communications in Algebra 26, 515-547, 1998.

[15] J.S.V. Symons, Some results concerning a transformation semigroup, Journal of the Australian Mathematical Society 19(series A), 413-425, 1975.

[16] K. Tinpun and J. Koppitz, Relative rank of the finite full transformation semigroup with restricted range, Acta Mathetmatica Universitatis Comenianae LXXXV, no. 2, 347-356, 2016.

[17] P. Zhao and V.H. Fernandes, The ranks of ideals in various transformation monoids, Communications in Algebra 43, 674-692, 2015. 